\title{
FLORA VASCULAR DEL HUMEDAL DE CARQUÍN - HUALMAY, HUAURA (LIMA, PERÚ)
}

\section{VASCULAR FLORA OF THE CARQUÍN-HUALMAY WETLAND (HUAURA, LIMA, PERU)}

\author{
Héctor Aponte ${ }^{1,2}$ y Asunción Cano ${ }^{2,3}$
}

\begin{abstract}
Resumen
Los humedales costeros junto a las lomas y tilandsiales constituyen un corredor biológico importante a lo largo de la costa peruano-chilena. El objetivo del presente trabajo es el de dar a conocer la flora vascular de un humedal ubicado entre los distritos de Carquín y Hualmay en la provincia de Huaura (Lima, Perú). Se reportan 41 especies de plantas vasculares, de las cuales 18 son Monocotiledóneas, 22 son Eudicotiledoneas y un Pteridófito; las familias más abundantes fueron Poaceae (7 especies) y Cyperaceae (5 especies). El 100\% corresponden a plantas de porte herbáceo o herbáceo-subfruticoso; $12 \%$ de las especies totales son plantas acuáticas flotantes o sumergidas. El 68\% de las especies (28 taxones) tienen reportadas al menos un uso, siendo más abundantes las de uso medicinal. Los resultados obtenidos muestran que este humedal posee una riqueza florística mayor a los registros más actuales de la Albufera de Medio Mundo (21 especies), Ventanilla (20 especies), Laguna El Paraíso (27) y Humedales de Puerto Viejo (32), pero menor al reportado para el Humedal Santa Rosa (67) y Pantanos de Villa (51). El análisis de similitud entre localidades muestra que este humedal guarda poca semejanza con los otros humedales de la costa $(0.31$ en promedio para el índice de Jaccard), reforzando la idea de que la diversidad beta de los humedales costeros es alta a nivel regional. Los datos encontrados para la cantidad de especies por hectárea indican que el humedal de Carquín-Hualmay, es el humedal que presenta el mayor número de especies de plantas vasculares por hectárea de toda la costa de Lima, con $3.4 \mathrm{sp}$./ha. Los resultados muestran que este humedal es un importante refugio de vida silvestre y debemos conservarlo como parte del corredor biológico de la costa de Lima.
\end{abstract}

Palabras clave: diversidad, flora, humedales, conservación.

\begin{abstract}
Coastal wetlands, along with the lomas formations and riverine ecosystems comprise an important biological corridor along the Peruvian-Chilean coast. The objective of this work was to study the vascular flora of a wetland located between the districts of Carquín and Hualmay in the province of Huaura (Lima, Peru). There are 41 species of vascular plants, of which 18 are Monocotyledons, 22 are Eudicotyledons and one Pteridophyte; the most abundant families were Poaceae ( 7 species) and Cyperaceae ( 5 species). The $100 \%$ correspond to herbaceous or subfruticous plants; $12 \%$ of the total species are floating or submerged aquatic plants. Sixty-eight percent of the species (28 taxa) have reported at least one use, being more abundant those of medicinal use. The results obtained show that this wetland has a floristic richness higher than the current records of the Albufera de Medio Mundo (21 species), Ventanilla (20 species), Laguna El Paraíso (27) and Humedales de Puerto Viejo (32), but lesser than those reported for Humedales de Santa Rosa (67) and Pantanos de Villa (51). The analysis of similarity between localities shows that this wetland bears little resemblance to the other coastal wetlands ( 0.31 on average for the Jaccard index), reinforcing the idea that the beta diversity of coastal wetlands is high at the regional level. The data for the number of species per hectare indicate that the Carquín-Hualmay wetland is the wetland with the highest number of vascular plant species per hectare along the entire coast of Lima, with 3.4 sp./ha. The results show that this wetland is an important refuge for wildlife and must be conserved it as part of the biological corridor of the coast of Lima.
\end{abstract}

Key words: diversity, flora, conservation, wetlands.

\section{Introducción.}

Los humedales de la costa central del Perú forman, junto con las lomas y los tilandsiales, un corredor de diversidad biológica en el desierto sudamericano (Aponte et al., 2012). Estudios recientes muestran que cada uno de los ecosistemas del corredor costero podría 
servir de refugio para especies de aves y plantas que lo utilizan (Aponte, 2016). Por esta razón, realizar estudios en cada uno de los componentes de este corredor, es importante para lograr entender el funcionamiento de estas islas como refugios de la diversidad biológica en esta región del planeta.

Los humedales costeros son ecosistemas que dependen íntimamente del agua proveniente de las cuencas de la vertiente occidental de los Andes; la misma puede llegar directamente a la costa, puede también filtrarse para formar parte de la napa freática, pasando inclusive por irrigaciones, formando los humedales costeros actuales (Aponte, 2017). Siendo la costa peruana una región altamente poblada, las poblaciones humanas se han encontrado con los humedales haciendo uso de sus múltiples bienes y servicios ecosistémicos (por ejemplo, el uso de las fibras del junco y la totora, y la provisión de un espacio para realizar actividades recreativas y turísticas; León et al., 1998; Carazas et al. 2016). Al mismo tiempo, los humedales han sufrido diferentes impactos antrópicos como el arrojo de desperdicios, la contaminación por silos, el constante impacto por incendios, el uso inadecuado de sus fuentes de agua y su reducción por el crecimiento urbano (Young, 1998; Aponte et al. 2017; Aponte \& Ramírez, 2014). La combinación de todas estas variables antrópicas, junto con las variables

a)

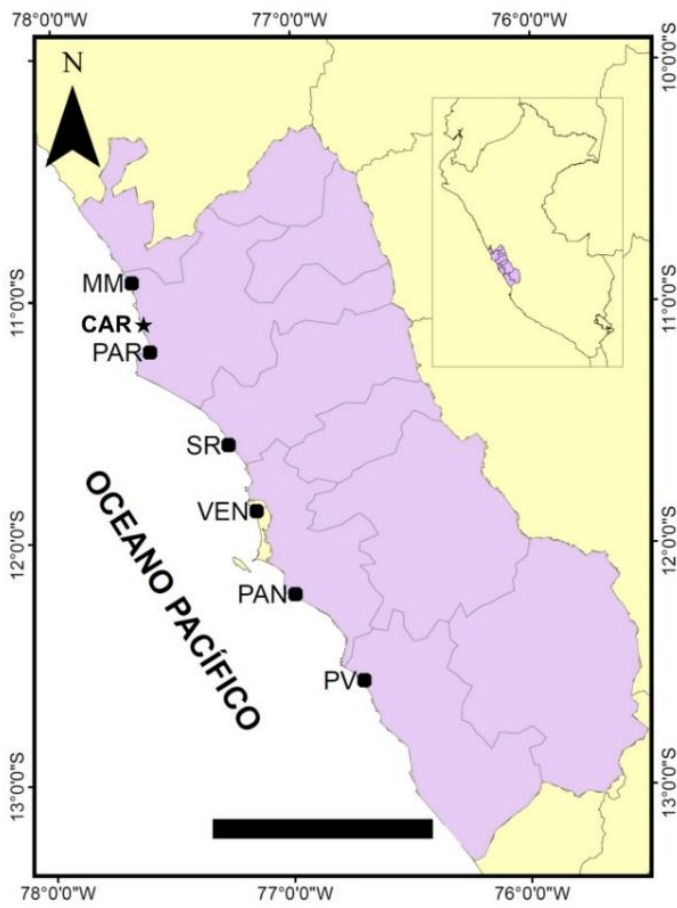

ambientales, han modelado las comunidades actuales presentes en estos ecosistemas.

Los intentos de documentar la composición de la flora vascular de los humedales costeros en el Perú, han mostrado que han existido y existen variaciones a lo largo del tiempo (Cano et al., 1993; León et al., 1995, 1998; Cano et al. 1998; Aponte \& Cano, 2013; entre otros). A la fecha se conocen un total de 122 especies de plantas vasculares como registro histórico, para seis humedales de la costa de Lima (Albufera de Medio Mundo, Laguna el Paraíso; Pantanos de Villa, Humedal de Santa Rosa, Humedales de Ventanilla y Humedales de Puerto Viejo) (Aponte \& Cano, 2013). De estas localidades, Pantanos de Villa es la que tiene la mayor diversidad histórica (71 especies históricamente, 51 en muestreos más recientes), mientras que los humedales de Santa Rosa albergan la mayor diversidad florística en el año en que se realizó el estudio (67 especies, año 2009). Un porcentaje considerable de plantas (45\%) corresponden a especies introducidas o invasoras, las cuales forman comunidades vegetales particulares; el $29 \%$ de las plantas no colectadas en los últimos años en los humedales de la región corresponden a especies acuáticas lo que refleja un impacto sobre los cuerpos de agua, por lo que podríamos indicar que las poblaciones de estas especies han pasado por procesos de extinción local (Aponte \& Ramírez, 2011; Aponte \& Cano, 2013). La similitud de su composición de flora vascular

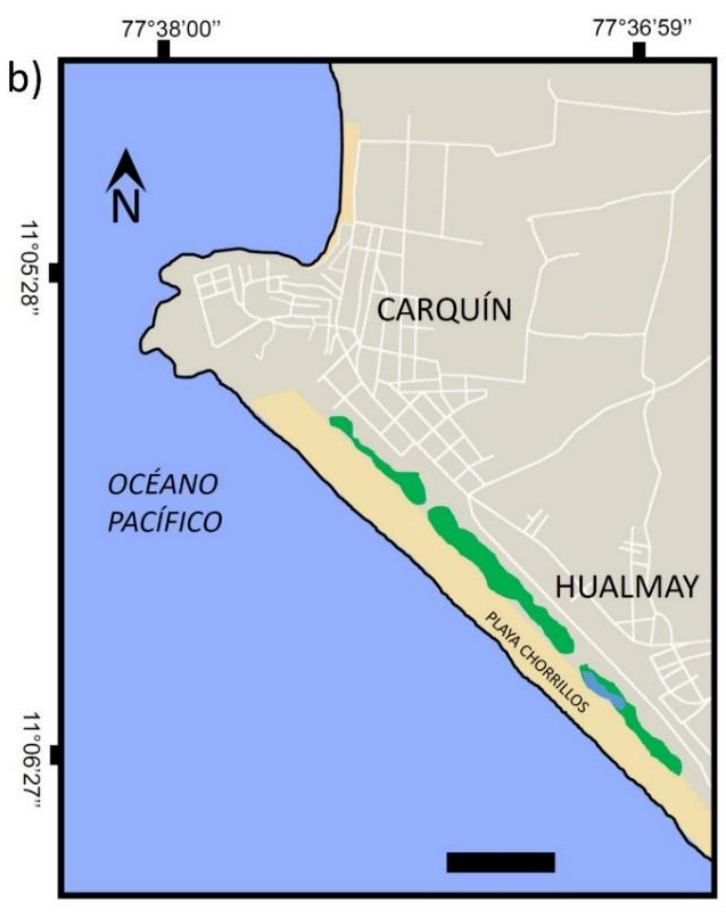

Figura 1. Área de estudio. a) Mapa de la costa de Lima mostrando los principales humedales costeros (MM=Albufera de Medio Mundo; PAR= Laguna El Paraíso; SR=Humedal Santa Rosa; VEN=Humedales de ventanilla; PAN=Pantanos de Villa; PV=Humedal de Puerto Viejo) y el humedal de Carquín - Hualmay (CAR); asimismo, se muestra en la esquina superior derecha el mapa del Perú; escala $=100 \mathrm{~km}$. b) Ubicación del Humedal de Carquín-Hualmay; escala $=200 \mathrm{~m}$. 
entre localidades es baja (31\%) lo que nos indica que la complementariedad entre localidades y la diversidad beta vegetal regional son altas (Cano et al., 1998; Aponte \& Ramirez, 2014). El estudio de nuevas localidades podría permitirnos conocer aún más el potencial que tienen estos ecosistemas para albergar la diversidad de especies en esta región.

El humedal de Carquín-Hualmay es un ecosistema ubicado entre los humedales de Paraíso y Medio Mundo (Figura 1a), los cuales forman también parte de este corredor costero. Hasta la fecha no se tiene publicaciones científicas sobre ningún componente biológico de este ecosistema, el cual está sufriendo una constante presión urbana. Conociendo su diversidad florística se pueden generar herramientas adecuadas para su gestión, conservación y monitoreo en el tiempo. El objetivo del presente trabajo fue el de conocer su composición florística del humedal de CarquínHualmay (Huaura; Lima-Perú).

\section{Área de estudio.}

El Humedal de Carquín-Hualmay se encuentra en la costa de los distritos de su mismo nombre (Carquín y Hualmay), pertenecientes a la provincia de Huaura

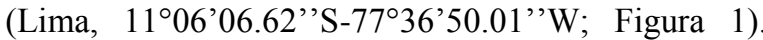
Este humedal se extiende a lo largo de dos kilómetros de costa (1 $940 \mathrm{~m}$ de largo y $60 \mathrm{~m}$ de ancho promedio, con 11.64 hectáreas) y se encuentra dividido por canales que conducen agua al humedal. El agua de estos canales se acumula en algunos puntos donde las poblaciones aledañas aprovechan este recurso para lavar sus prendas de vestir. El humedal posee una laguna de 200 metros de largo y otros pequeños espejos de agua; todos los cuerpos de agua varían de tamaño a lo largo del año. Estos espacios se encuentran rodeados de juncales, gramadales y comunidades mixtas (estas últimas presentan un porte bajo, menor a $15 \mathrm{~cm}$.). Durante la evaluación se apreció ganado vacuno en el área de estudio. El humedal limita por el lado este con una vía por donde circulan autos en la cual pueden apreciarse zonas de acopio de residuos sólidos.

\section{Métodos.}

Colecta de especies vegetales.

Se realizaron cuatro colectas trimestrales en los diferentes hábitats del humedal, de acuerdo a técnicas estandarizadas (Bridson \& Forman, 1998) y anotando las especies predominantes de los hábitats encontrados. Para esta colecta se solicitó el permiso respectivo a la autoridad competente (SERFOR/DGGSPFFS Autorización $\mathrm{N}^{\circ}$ AUT-IFL-2016-048). Se tomaron registro de las formas de crecimiento (Wittaker, 1975). Asimismo, se registraron las coordenadas geográficas y altitudes mediante un receptor GPS. Los ejemplares recolectados se depositaron en el Herbario San Marcos (USM). Las determinaciones taxonómicas fueron realizadas en el Laboratorio de Florística del Museo de Historia Natural UNMSM, empleando claves y descripciones publicadas en literatura especializada (León, 1993; Sagástegui \& Leiva, 1993), listados previos en otros humedales de Lima (Cano et al., 1998; La Torre \& Aponte, 2011; Aponte \& Cano, 2013) además de consultas a especialistas. Las muestras fueron corroboradas con especímenes depositados en USM.

La información de usos y su clasificación como especies introducidas se obtuvieron del catálogo del plantas útiles de la Amazonía del Perú (Rutter \& Shanks, 1990), de trabajos sobre plantas útiles de los humedales de Lima (León et al., 1998) y de la base de datos del Germplasm Resource Information Center (USDA et al., 2017). Se consideró como planta con potencial invasivo a aquella reportada como mala hierba (según USDA et al., 2017).

Análisis comparativo con otros humedales de Lima.

Se realizó un análisis de similitud de especies de plantas vasculares entre los humedales de la costa central del Perú, utilizando la última publicación de cada humedal como dato actual (para Pantanos de Villa se utilizó (Ramirez \& Cano, 2010), para los humedales de Puerto Viejo, Santa Rosa, Medio Mundo y la Laguna El Paraíso se utilizó (Aponte \& Cano, 2013), para los Humedales de Ventanilla se utilizó Aponte \& Ramirez (2014). Las comparaciones se hicieron utilizando el índice de Jaccard (Ij) cuya fórmula se encuentra representada en la ecuación:

$$
I j=C /(C+N C)
$$

Donde $\mathrm{C}$ son el número de especies comunes y $\mathrm{NC}$ es el número de especies no comunes. Esta evaluación fue acompañada de un análisis de agrupamiento con el método de comparación por pares calculando el Coeficiente Cofenético; este coeficiente mide cuán robusto es el agrupamiento y varía entre cero y uno. El promedio de los Ij obtenidos en la comparación por pares fue utilizado como un indicador de la diversidad Beta regional. Todos estos análisis fueron realizados utilizando el software PAST V. 2.8 (Hammer et al., 2001).

Adicionalmente se comparó la riqueza del humedal de Carquín-Hualmay con la de los otros humedales, así como la cantidad de especies por hectárea (sp/ha). En este caso se utilizó también la última publicación de cada humedal como dato actual de su riqueza y de su extensión (referencias mencionadas en el párrafo anterior).

\section{Resultados.}

Durante el periodo de estudio fueron reportadas un total de 41 especies de plantas vasculares, de las cuales 18 son monocotiledóneas, 22 son eudicotiledóneas y un pteridófito; las familias más abundantes fueron Poaceae (7 especies) y Cyperaceae (5 especies) (Tabla 1). El $100 \%$ corresponden a plantas de porte herbáceo o herbáceo-subfruticoso; $12 \%$ de las especies totales son 
plantas acuáticas flotantes o sumergidas, las que fueron encontradas principalmente en los canales (como en el caso de Lemna minuta Kunth "lenteja de agua", Wolffia columbiana H. Karst. y Eichhornia crassipes (Mart.) Solms_Laubach "jacinto de agua") o en los bordes de las lagunas (como en el caso de Potamogeton striatus Ruiz \& Pav.). Los juncales de este humedal estuvieron caracterizados por la predominancia de Schoenoplectus americanus (Pers.) Volkart ex Schinz \& R. Keller "junco"; los gramadales tuvieron diferentemente la predominancia de Distichlis spicata (L.) Greene, Paspalum vaginatum Swartz. o Sporobolus virginicus (L.) Kunth; las comunidades mixtas estuvieron compuestas por mezclas de especies de los pastizales, juncales, especies de porte bajo como Eleocharis geniculata (L.) Roem. \& Schult. y Triglochin striatum Ruiz \& Pav., y por especies herbáceas como Ludwigia peploides (Kunth) P.H. Raven.

El $68 \%$ de las especies (28 taxones) tienen reportados al menos un uso, siendo más abundantes las de uso medicinal. El $36 \%$ del total de especies reportadas (15 especies) tienen potencial invasivo. Durante el muestreo se encontraron también especies cultivadas como Cucumis sp. y Physalis peruviana L.; estas últimas no han sido consideradas en el listado.

Análisis comparativo.

La Tabla 2 y Figura 2 muestran los resultados de la comparación por pares del Índice de Jaccard (Ij) entre los humedales costeros. El Humedal de CarquínHualmay presenta una mayor similitud con los Pantanos de Villa $(\mathrm{Ij}=0.46)$ y con los humedales de Puerto Viejo y Santa Rosa (ambos con un $\mathrm{Ij}=0.35$ ). El análisis de agrupamiento coloca al Humedal de Carquín-Hualmay en un grupo separado de Pantanos de Villa y los humedales de Santa Rosa (Figura 2, Coeficiente cofenético $=0.84)$. El promedio de los $\mathrm{Ij}$ obtenidos en la comparación por pares fue de 0.31 . Los resultados obtenidos muestran que este humedal posee una riqueza florística mayor a los registros actuales de la Albufera de Medio Mundo (21 especies), Ventanilla (20 especies), Laguna El Paraíso (27) y Humedales de Puerto Viejo (32), pero menor al reportado para el Humedal Santa Rosa (67) y Pantanos de Villa (51).

Los datos encontrados para la cantidad de especies por hectárea indican que el humedal de CarquínHualmay, es el humedal que presenta el mayor número de especies de plantas vasculares por hectárea con 3.4 sp/ha, seguido del Humedal Santa Rosa (2.1 sp/ha), los humedales de Puerto Viejo (0.2 sp/ha), Pantanos de Villa (0.2 sp/ha), la Albufera de Medio Mundo (0.1 $\mathrm{sp} / \mathrm{ha}$ ), ACR Humedales de Ventanilla (con $0.1 \mathrm{sp} / \mathrm{ha}$ ) y la Laguna El Paraíso (0.03 sp/ha).

\section{Discusión.}

Por lo encontrado en el presente trabajo, se muestra la importancia de este humedal en la costa peruana. Los datos obtenidos muestran que este ecosistema es el tercer humedal de mayor riqueza florística de la costa

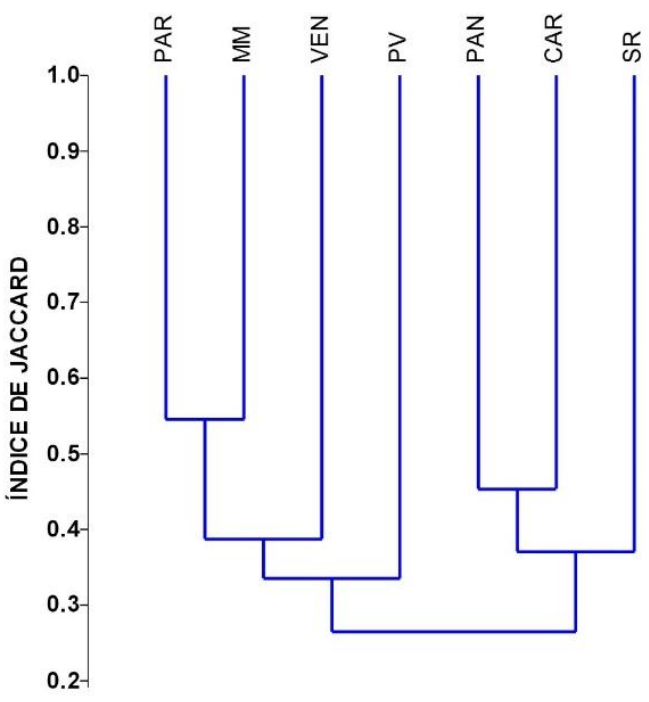

Figura 2. Análisis de agrupamiento entre los humedales de la costa central del Perú, donde se incluye el humedal de Carquín-Hualmay (CAR). Coeficiente cofenético $=0.84$. PAR=Laguna $\mathrm{El}$ Paraíso; MM=Albufera de Medio Mundo; VEN=Humedales de Ventanilla; $\mathrm{PV}=$ Humedales de Puerto Viejo; PAN=Pantanos de Villa; CAR=Humedal de Carquín-Hualmay; $\mathrm{SR}=$ Humedal Santa Rosa.

de Lima y el que tiene mayor cantidad de especies por unidad de área, lo que lo convierte en un humedal muy importante como refugio de flora vascular en la costa desértica del Perú. Hasta la fecha son escasas las publicaciones científicas sobre este ecosistema (siendo este el primer artículo científico sobre el ecosistema en estudio), por lo que se desconoce el potencial completo que tiene este humedal como refugio de aves, mamíferos, reptiles, arácnidos, insectos y organismos microscópicos, los cuales son muy importantes en los humedales costeros y que han mostrado tener una alta riqueza cuando han sido evaluados (Icochea, 1998; Guillén, 2002; Paredes, 2010; Alarcón \& Iannacone, 2014; Guillén et al., 2015; Pacheco et al., 2015). Es necesario continuar con los estudios de estos grupos en esta localidad a fin de tener una visión real de la diversidad que se alberga en el humedal de CarquínHualmay.

El análisis comparativo refuerza la idea de que la diversidad beta en los humedales costeros de Lima es alta (la similitud promedio entre humedales se encuentra alrededor del 30\%). Por esta razón, es fundamental conservar inclusive aquellos humedales pequeños como la localidad del presente estudio, ya que la complementariedad entre las zonas evaluadas es alta. Cinco especies (Bidens pilosa L.; Lepidium didymum L.; Ricinus communis L.; Setaria verticillata (L.) P. Beauv.; Sonchus oleraceus L.; Spilanthes urens Jacq.) son reportadas por primera vez para los humedales 
costeros de Lima. Todas ellas, salvo S. urens, tienen potencial invasivo (Tabla 1); estas especies pueden haber llegado al humedal por medio de los canales que ingresan al humedal, o fruto de la interacción con las poblaciones aledañas. De la misma forma, las especies cultivadas no incluidas en el listado, son muestra de la interacción con las poblaciones humanas. Durante el muestreo fue posible apreciar la presencia de ganado vacuno en el área de estudio, factor que pueda también haber influido en la riqueza de flora vascular del área (en este caso, favoreciendo el establecimiento de pequeñas especies, fenómeno apreciado en el Humedal Santa Rosa; Aponte \& Ramírez, 2011). El uso del agua de los canales para lavandería puede afectar positiva (aumentando los nutrientes y permitiendo el crecimiento) o negativamente a la flora del lugar (modificando las interacciones entre especies, favoreciendo a aquellas especies que son capaces de asimilar rápidamente los fosfatos). Mayores estudios son necesarios para tener una idea clara de cómo todos estos impactos pueden estar afectando a los diferentes componentes biológicos de este humedal. A ello debemos sumar el control del impacto humano y la adecuada gestión del espacio; retos identificados anteriormente para los humedales costeros de Lima (Aponte et al., 2012) que deben de tomarse en cuenta para la mejor protección de este ecosistema.

La composición del paisaje encontrado en el humedal de Carquín-Hualmay es fruto de las interacciones de todas las variables que convergen en este sector de la costa peruana. Con los datos obtenidos en el presente trabajo se sientan las bases para que futuras investigaciones puedan hacer un seguimiento del humedal a lo largo del tiempo; asimismo, se provee de un listado de plantas vasculares y sus usos que puede ser utilizado por los gestores ambientales (como la Gerencia de Medio Ambiente de la Región Lima y la Gerencia de Medio Ambiente de la Municipalidad de la Caleta de Carquín) para justificar la protección y cuidado de este sector marino-costero.

\section{Conclusiones.}

El presente estudio reporta 41 especies de plantas vasculares para el humedal de Carquín-Hualmay; con este registro, este humedal se ubica dentro de los tres humedales costeros más ricos en plantas vasculares de toda la Región Lima y en el primer lugar por el número de especies por unidad de área. Por estas razones, este humedal debe protegerse y estudiarse, a fin de conocer el verdadero potencial de este ecosistema como refugio de vida en el desierto peruano.

\section{Agradecimientos.}

Quedamos plenamente agradecidos a la Municipalidad de Carquín, quien nos brindó apoyo logístico para la realización del presente estudio. Asimismo, agradecemos a la Dirección General Forestal y de Fauna Silvestre del SERFOR (Ministerio de Agricultura) por las facilidades brindadas para la obtención del permiso de colecta. El presente trabajo contó con el financiamiento del Instituto Peruano para la Sostenibilidad y el Desarrollo. Finalmente, agradecemos a los revisores del presente artículo quienes han permitido mejorarlo sustancialmente.

\section{Literatura citada.}

Alarcón G. \& Iannacone J. 2014. Artropofauna terrestre asociada a formaciones vegetales en el Refugio de Vida Silvestre Pantanos De Villa, Lima, Perú. The Biologist (Lima), 12, 253-274.

Aponte H. \& Cano A. 2013. Estudio florístico comparativo de seis humedales de la costa central del Perú: Actualización y nuevos retos para su conservación. Revista Latinoamericana de Conservación, 3(2), 15-27.

Aponte H. \& Ramirez D.W. 2014. Riqueza florística y estado de conservación del Área de Conservación Regional Humedales de Ventanilla (Callao, Perú). Biologist (Lima), 12(2), 270-282.

Aponte H. \& Ramírez W. 2011. Los Humedales de La Costa central del Perú: Comunidades Vegetales y Conservación. Ecología Aplicada, 10(1), 31-39.

Aponte H. 2016. Nuevo registro de flora para las Lomas de Lachay: Primer reporte de Lemna minuta Kunth (Araceae). Ecología Aplicada, 15(1), 57-60.

Aponte H. 2017. Humedales de la Costa central del Perú: Un diagnóstico de los humedales de Santa Rosa, laguna El Paraíso y Albufera de Medio Mundo. Cooperacción 114pp.

Aponte H., Jiménez R. \& Alcántara B. 2012. Challenges for management and conservation of Santa Rosa Wetland (Lima - Peru). Cientifica, 9(3), 257-264.

Aponte H., Ramírez D.W. \& Vargas R. 2017. Primeros estadios en la regeneración natural de la vegetación postincendio en los Humedales de Ventanilla (Lima-Perú). Ecología Aplicada 16(1) pp 23-30.

Bridson D.M. \& Forman L. 1998. The Herbarium Handbook, 3rd ed. Royal Botanic Gardens, Kew.

Cano A., La Torre M.I., León B., Roque J. \& Arakaki M. 1998. Estudio comparativo de la Flora vascular de los Principales Humedales de las Zona Costera del Departamento de Lima, Perú. Los Pantanos de Villa: Biología y Conservación (eds A. Cano \& Young), pp. 181-190. Universidad Nacional Mayor de San Marcos, Lima - Perú.

Cano A., León B. \& Young K. 1993. Plantas vasculares de los Pantanos de Villa, Lima. Las Plantas Acuáticas en las Aguas Continentales del Perú (eds F. Kahn, B. León, \& K. Young), pp. 177-207. Instituto Francés de Estudios Andinos.

Carazas N., Gil F., Aponte H., Velásquez W., Paucar M., Salazar R. \& Zárate R. 2016. Área de Conservación Regional Humedales de Ventanilla: Estado actual del conocimiento biológico y turístico. Novum Otium 2(1). 923.

Guillén G. 2002. Diversidad Protozoológica de Los Pantanos de Villa. Tesis para optar por el título profesional de Biólogo con mención en Zoología, Universidad Nacional Mayor de San Marcos., Lima - Perú.

Guillén G., Aponte H., Bacigalupo X. \& Rodriguez R. 2015. Protozoarios de vida libre del Área de Conservación Regional Humedales de Ventanilla (Callao - Perú) en el 
período septiembre 2011 - enero 2012. Científica, 12(1), 61-69.

Hammer Ø., Harper, D.A.T. \& Rayan P.D. 2001. PAST: Paleontological statistics software package for education and data analysis. Palaeontologia Electronica, 4(1), 9.

Icochea J. 1998. Lista Roja preliminar de los anfibios y reptiles amenazados del departamento de Lima. Los Pantanos de Villa: Biología y Conservación 11., pp. 217 219. Universidad Nacional Mayor de San Marcos, Lima, Perú.

La Torre M.I. \& Aponte H. 2011. Flora vascular y vegetación de los humedales de Puerto Viejo. Revista Peruana de Biología, 16, 215-217.

León B. 1993. Catálogo anotado de las fanerógamas acuáticas del Perú. Las Plantas Vasculares en las Aguas Continentales del Perú Travaux de l'Institut Francais d'Etudes Andines. (eds F. Kahn, B. León, \& K. Young), pp. 11-128. IFEA (Institut Francais d'Etudes Andines), Lima-Peru.

León B., Cano A. \& Young K. 1995. La flora vascular de los Pantanos de Villa, Lima, Perú: Adiciones y guía para las especies comunes. Publicaciones del Museo de Historia Natural-UNMSM. (B), 38, 1-39.

León B., Cano A. \& Young K. 1998. Uso Actual de la Flora y Vegetación en los Humedales de la Costa Central del Perú. Los Pantanos de Villa: Biología y Conservación Serie de Divulgación $N^{\circ} 11$. (eds A. Cano \& K. Young), pp. 191-104. Universidad Nacional Mayor de San Marcos, Lima - Perú.

Pacheco V., Zevallos A., Cervantes K., Pacheco J. \& Salvador J. 2015. Mamíferos del Refugio de Vida Silvestre Los pantanos de Villa, Lima, Perú. Cientifica, 12(1), 26-41.

Paredes W. 2010. Diversidad y Variación Espacio-Temporal de Las Comunidades de Arañas En La Zona Reservada de Pantanos de Villa, Lima, Perú. Tesis para optar por el grado de bachiller en Ciencias Biológicas, Universidad Nacional Mayor de San Marcos, Lima - Perú.

Ramirez D. \& Cano A. 2010. Estado de la diversidad de la flora vascular de los Pantanos de Villa (Lima - Perú). Revista Peruana de Biología, 17, 111-114.

Rutter R.A. \& Shanks A. 1990. Catálogo de plantas utiles de la Amazonía Peruana. Ministerio de Educación.

Sagástegui A. \& Leiva S. 1993. Flora invasora de los cultivos del Peru. CONCYTEC.

USDA, ARS, National Genetic Resources Program. 2017. Germplasm Resources Information Network - (GRIN) [On Line Data Base]. Disponible en: https://npgsweb.arsgrin.gov/gringlobal/taxon/taxonomysearcheco.aspx

Wittaker R.H. 1975. Comunities and Ecosystems. Macmillan Publishing Co. Inc.

Young K. 1998. El Ecosistema. Los Pantanos de Villa: Biología y Conservación Serie de Divulgación $\mathrm{N}^{\circ} 11$. (eds. A. Cano \& K. Young), pp. 3-20. Universidad Nacional Mayor de San Marcos, Lima - Perú.

Tabla 1. Familias y especies de flora vascular del Humedal de Carquín-Hualmay.

\begin{tabular}{|c|c|c|c|c|c|c|c|}
\hline FAMILIA & Especie + Autor & AC & USDA & $\begin{array}{l}\text { León } \\
\text { et al. }\end{array}$ & Rutter & PI & $\begin{array}{c}\text { Número } \\
\text { H } \\
\text { Aponte }\end{array}$ \\
\hline AIZOACEAE & Sesuvium portulacastrum (L.) L. & & OR, RV & & & & 308 \\
\hline AMARANTHACEAE & $\begin{array}{l}\text { Alternanthera halimifolia (Lam.) Standl. ex } \\
\text { Pittier }\end{array}$ & & & & MD & & 303 \\
\hline AMARANTHACEAE & $\begin{array}{l}\text { Dysphania ambrosioides (L.) Mosyaquin } \\
\text { \&Clemants }\end{array}$ & & $\begin{array}{l}\text { AA, } \\
\text { MAT, } \\
\text { MD,VEN }\end{array}$ & & MD & & 312 \\
\hline ARACEAE & Colocasia esculenta (L.) Schott & & $\begin{array}{c}\text { AF, OR, } \\
\text { AH, FO, } \\
\text { GEN, } \\
\text { MD, } \\
\text { VEN }\end{array}$ & & $\mathrm{AH}$ & $\mathrm{X}$ & 321,277 \\
\hline ARACEAE & Lemna minuta Kunth. & $\mathrm{AF}$ & & $\begin{array}{l}\text { FO, } \\
\text { IC, } \\
\text { RM }\end{array}$ & & & 275 \\
\hline ARACEAE & Wolffia columbiana $\mathrm{H}$. Karst. & $\mathrm{AF}$ & & & & & 295 \\
\hline ARALIACEAE & Hydrocotyle bonariensis Lam. & & & & & & 297,320 \\
\hline ARALIACEAE & Hydrocotyle ranunculoides L.f. & & & & & $\mathrm{X}$ & 280,316 \\
\hline ASTERACEAE & Bidens pilosa $\mathrm{L}$ & & $\begin{array}{l}\mathrm{CP}, \mathrm{CH} \\
\mathrm{MD}\end{array}$ & & MD & $\mathrm{X}$ & 311 \\
\hline ASTERACEAE & Enydra sessilifolia (Ruiz \& Pav.) Cabrera & & & & & & 286 \\
\hline ASTERACEAE & Sonchus oleraceus L. & & MD & & & $\mathrm{X}$ & 314 \\
\hline ASTERACEAE & Spilanthes urens Jacq. & & & & & & 300 \\
\hline AZOLLACEAE & Azolla filiculoides Lam. & $\mathrm{AF}$ & $\begin{array}{l}\text { OR, SU, } \\
\text { FO }\end{array}$ & $\begin{array}{l}\mathrm{IC}, \\
\mathrm{RM}, \\
\mathrm{AB}\end{array}$ & & $\mathrm{X}$ & 294 \\
\hline BORAGINACEAE & Heliotropium curassavicum L. & & $\begin{array}{l}\text { MD, } \\
\text { VEN }\end{array}$ & & MD & & 289 \\
\hline BRASSICACEAE & Lepidium didymum $\mathrm{L}$. & & & & & $\mathrm{X}$ & 317 \\
\hline CYPERACEAE & Cyperus laevigatus $\mathrm{L}$. & & $\begin{array}{l}\text { FO, } \\
\text { MAT }\end{array}$ & & & & 283 \\
\hline
\end{tabular}


Tabla 1. Familias y especies de flora vascular del Humedal de Carquín-Hualmay.

\begin{tabular}{|c|c|c|c|c|c|c|c|}
\hline FAMILIA & Especie + Autor & $\mathbf{A C}$ & USDA & $\begin{array}{l}\text { León } \\
\text { et al. }\end{array}$ & Rutter & PI & $\begin{array}{c}\text { Número } \\
\text { H } \\
\text { Aponte }\end{array}$ \\
\hline CYPERACEAE & Eleocharis geniculata (L.) Roem. \& Schult. & & & & & & 285 \\
\hline CYPERACEAE & $\begin{array}{l}\text { Schoenoplectus americanus (Pers.) Volkart } \\
\text { ex Schinz \& R. Keller }\end{array}$ & & $\begin{array}{l}\text { CE, } \\
\text { MAT, } \\
\text { VEN }\end{array}$ & MAT & & & 274 \\
\hline CYPERACEAE & $\begin{array}{l}\text { Schoenoplectus californicus (C.A. Mey.) } \\
\text { Soják }\end{array}$ & & $\begin{array}{l}\text { CE, } \\
\text { MAT }\end{array}$ & MAT & & $\mathrm{X}$ & 281,315 \\
\hline CYPERACEAE & Torulinium odoratum (L.) S.S. Hooper & & & & & & 284 \\
\hline EUPHORBIACEAE & Ricinus communis $\mathrm{L}$. & & $\begin{array}{l}\text { AA, OR, } \\
\text { FO, MAT } \\
\text { VEN }\end{array}$ & $\begin{array}{l}\text { COM, } \\
\Gamma, \mathrm{MD}, \\
\mathrm{N}\end{array}$ & $\begin{array}{l}\text { COM, } \\
\text { MD }\end{array}$ & $\mathrm{X}$ & 318 \\
\hline FABACEAE & Vigna luteola (Jacq.) Benth. & & FO, GEN & & & & 304 \\
\hline JUNCAGINACEAE & Triglochin striatum Ruiz \& Pav. & & & & & & 273 \\
\hline ONAGRACEAE & Ludwigia peploides (Kunth) P.H. Raven & & & & & $\mathrm{X}$ & 287,309 \\
\hline PLANTAGINACEAE & Bacopa monnieri (L.) Wettst. & & OR, MD & & & & 282 \\
\hline PLANTAGINACEAE & Plantago major $\mathrm{L}$. & & MD & & MD & $\mathrm{X}$ & 279 \\
\hline POACEAE & Cynodon dactylon (L.) Pers. & & $\begin{array}{c}\mathrm{FO}, \mathrm{CE}, \\
\mathrm{MED}, \\
\mathrm{VEN}\end{array}$ & $\begin{array}{l}\text { FO, } \\
\mathrm{CE}, \\
\mathrm{MD}\end{array}$ & MD & & 290 \\
\hline POACEAE & Distichlis spicata $(\mathrm{L}$.$) Greene$ & & CE & & & & 299 \\
\hline POACEAE & Paspalidium geminatum (Forssk.) Stapf & & FO & IC & & & 292 \\
\hline POACEAE & Paspalum vaginatum Swartz. & & CE, RV & $\begin{array}{l}\text { FO } \\
\text { CE }\end{array}$ & & & 323 \\
\hline POACEAE & Polypogon viridis (Gouan) Breistr. & & & & & $\mathrm{X}$ & 291 \\
\hline POACEAE & Setaria verticillata (L.) P. Beauv. & & $\mathrm{FO}$ & & & $\mathrm{X}$ & 313 \\
\hline POACEAE & Sporobolus virginicus (L.) Kunth & & CE, FO & & & $\mathrm{X}$ & 296 \\
\hline POLYGONACEAE & Polygonum hydropiperoides Michx. & & & & & & 319 \\
\hline POLYGONACEAE & Rumex crispus $\mathrm{L}$. & & $\begin{array}{l}\text { MD, } \\
\text { VEN }\end{array}$ & & & $\mathrm{X}$ & 302 \\
\hline PONTEDERIACEAE & Eichhornia crassipes (Mart.) Solms_Laubach & $\mathrm{AF}$ & $\begin{array}{l}\text { SU, OR, } \\
\text { FO }\end{array}$ & $\begin{array}{r}\text { FO, IC } \\
\text { O }\end{array}$ & R & & 276 \\
\hline POTAMOGENONACEAE & Potamogeton striatus Ruiz \& Pav. & AS & & & & & 301 \\
\hline SOLANACEAE & Solanum americanum Mill. & & $\mathrm{AH}$ & & & $\mathrm{X}$ & 306 \\
\hline SOLANACEAE & Solanum pimpinellifolium $\mathrm{L}$. & & $\begin{array}{l}\text { AH, } \\
\text { GEN }\end{array}$ & & & & 305 \\
\hline TYPHACEAE & Typha domingensis Pers. & & $\begin{array}{l}\text { MD, } \\
\text { MAT }\end{array}$ & $\mathrm{FO} / \mathrm{IC}$ & & & 293,322 \\
\hline VERBENACEAE & Lippia nodiflora (L.) Michx & & & & & & 278 \\
\hline
\end{tabular}

Se indica si son acuáticas (AC) flotantes (AF) o sumergidas (AS). Se muestran los usos encontrados según las diferentes referencias bibliográficas utilizadas (USDA, 2017; León et al., 1998; Rutter \& Shanks, 1990). Se indica si la especie tiene potencial invasivo (PI) según USDA (2017) y su número de colecta. -=no presentó datos en bibliografía consultada. Para los usos OR=Ornamental; RV=Revegetación; $\mathrm{SU}=$ Mejoramiento de suelo; $\mathrm{MED=Medicinal;} \mathrm{AF=Agroforestería;}$ $\mathrm{GEN}=$ Fuente de genes; VEN=venenosa; $\mathrm{AH}=\mathrm{Alimento}$ humano; $\mathrm{AB}=\mathrm{Abono} ; \mathrm{RM}=$ Remediación; $\mathrm{CE}=\mathrm{Control}$ de la erosión; $\mathrm{AA}=$ Aditivo para alimentos; $\mathrm{COM}=$ Combustible; $\mathrm{FO}=$ Forraje o alimento animal; IC=Indicador de Contaminación.

Tabla 2. Análisis comparativo de similitud entre localidades utilizando el Índice de Jaccard.

\begin{tabular}{|c|c|c|c|c|c|c|}
\hline & PAN & SR & PAR & MM & VEN & CAR \\
\hline $\mathrm{PV}$ & 0.29688 & 0.20732 & 0.33333 & 0.39474 & 0.30233 & 0.35185 \\
\hline PAN & & 0.38824 & 0.31667 & 0.28571 & 0.22951 & 0.46032 \\
\hline SR & & & 0.26667 & 0.18919 & 0.16667 & 0.35000 \\
\hline PAR & & & & 0.53125 & 0.33333 & 0.32692 \\
\hline MM & & & & & 0.40625 & 0.29167 \\
\hline VEN & & & & & & 0.20370 \\
\hline
\end{tabular}




\footnotetext{
${ }^{1}$ Facultad de Ciencias Veterinarias y Biológicas. Carrera de Biología Marina. Universidad Científica del Sur. Av. Antigua Carretera Panamericana Sur km 19 Villa El Salvador. - Lima 42, Perú. hectoaponte@ gmail.com.

${ }^{2}$ Laboratorio de Florística, departamento de Dicotiledóneas, Museo de Historia Natural, Universidad Nacional Mayor de San Marcos. Avenida Arenales 1256, Jesús María - Lima. Apartado 14-0434, Lima 14, Perú.

${ }^{3}$ Instituto de Investigación de Ciencias Biológicas Antonio Raimondi (ICBAR). UNMSM.
} 